\title{
Detection of Candida auris and its antifungal susceptibility: first report from Bangladesh
}

\author{
Subarna Dutta ${ }^{1}$, Md. Hasibur Rahman ${ }^{2}$, Kazi Shakhawath Hossain ${ }^{3}$, Jalaluddin Ashraful Haq ${ }^{4}$ \\ ${ }^{1}$ Department of Microbiology, BIRDEM General Hospital, Shahbag, Dhaka, Bangladesh, \\ ${ }^{2}$ Department of Microbiology, Jahangirnagar University, Savar, Dhaka, Bangladesh, \\ ${ }^{3}$ Department of Botany, Faculty of Life \& Earth Science, Jagannath University, Dhaka, Bangladesh, \\ ${ }^{4}$ Department of Microbiology, Ibrahim Medical College, Shahbag Avenue, Dhaka, Bangladesh
}

\begin{abstract}
Background and objectives: Candida auris is an emerging multidrug-resistant fungal pathogen that has been associated with nosocomial infections with a high mortality. The organism has been reported from several countries of the world except Bangladesh. The present study describes the presence of $C$. auris in clinical samples obtained from a large hospital of Dhaka city, Bangladesh.
\end{abstract}

Materials and methods: The A total of 100 Candida species isolated from different clinical samples were purposively included in the present study. Samples were obtained from patients attending a 750 bed hospital of Dhaka city. C. auris was identified by growth characteristics, biochemical and carbohydrate assimilation test and further confirmed by polymerase chain reaction and sequencing using ITS1 and ITS2 targeting the conserved regions of 5.8S rRNA. Antifungal susceptibility of identified $C$. auris was performed by disk diffusion and minimum inhibitory concentration (MIC) methods.

Results: Out of 100 Candida sp. tested, 21 isolates were identified as C. auris. Of the 21 C. auris, 14 (66.7\%) were isolated from blood samples and the remaining 7 (33.4\%) were from urine. Most of the $C$. auris isolated were from patients admitted in intensive care units. Out of $21 C$. auris, 17 (81.0\%), 7 (33.3\%) and 3 (14.3\%) were sensitive to amphotericin B, fluconazole and voriconazole respectively by disk diffusion method. Out of 14 fluconazole resistant isolates, 5 were susceptible dose-dependent (SS-D) by MIC method.

Conclusion: The present study is the first report demonstrating the presence of $C$. auris in clinical samples obtained from a large hospital of Bangladesh. Majority of isolates showed resistance to fluconazole and variable susceptibility to other antifungal agents. Further study is suggested to find its true magnitude and its susceptibility pattern to a range of antifungal agents.

IMC J Med Sci 2019; 13(2): 003. EPub date: 05 August 2019

\section{Introduction}

Candida is now recognized as a major agent of hospital-acquired infection [1]. Although, most infections are attributed to $C$. albicans, the shift towards treatment resistant non-albicans Candida (NAC) species is increasingly evident in recent years
$[2,3]$. C. auris is an emerging NAC species which is first reported in Japan in 2009 [4]. Studies from several countries have documented that $C$. auris is causing severe illness in hospitalized patients and difficult to control hospital outbreaks $[5,6]$. The organism is extremely transmissible between patients, 
inter healthcare facilities and from contaminated environments [7-9]. Infection by $C$. auris requires proper attention as it shows resistance to many commonly used antifungal agents [10-12]. A study from India has reported that $90 \%, 15 \%$ and $8 \%$ of $C$. auris isolated between 2009 and 2017 were resistant to fluconazole, voriconazole and amphotericin B, respectively [12].

Identification of $C$. auris is not usually done in routine microbiology practice due to lack of awareness about the organism and limited laboratory facilities. Moreover, $C$. auris is often difficult to differentiate from other NAC species in laboratories with limited biochemical tests. No study has yet been done in Bangladesh with regard to the detection and antifungal susceptibility of $C$. auris.

The present study investigated the presence of $C$. auris in different clinical samples obtained from patients attending a hospital of Dhaka city. Its susceptibility to common antifungal agents was also determined.

\section{Materials and methods}

Study samples and place: The study was carried out at a 750 bed-hospital of Dhaka city over a period of one year. A total of 100 Candida species isolated from different clinical samples were purposively included in the present study for detail species identification. Clinical samples included urine, blood, sputum, pus, high vaginal swab and body fluid from patients admitted in wards, intensive care unit (ICU) and neonatal intensive care unit (NICU).

Isolation and identification of $\boldsymbol{C}$. auris: All clinical samples were inoculated on the Sabouraud Dextrose Agar (SDA) media. Phenotypic features of $C$. auris were identified by wet film, (oval or round shape yeast or budding yeast cell), Gram staining (Gram positive yeast cell), and incubation at $37-42^{\circ} \mathrm{C}$ temperature $[13,14$,]. Carbohydrate assimilation test was performed as described earlier [15]. C. auris identified by growth characteristics, biochemical reactions and carbohydrate assimilation tests were further confirmed by polymerase chain reaction and sequencing using ITS1 and ITS2 targeting the conserved regions of 5.8S rRNA [16].The purified PCR product was sent to McLab, California, USA for sequencing. The sequence was used as probes in $\mathrm{NCBI}$ blast search database in order to retrieve similar sequences.

\section{Determination of antifungal susceptibility}

a. Disk diffusion method (DDM): The isolates were tested for susceptibility to amphotericin B $(10 \mu \mathrm{g})$, fluconazole $(25 \mu \mathrm{g})$ and voriconazole $(1 \mu \mathrm{g})$ by disk diffusion method as described in NCCLS manual M44A, 2004 [17]. The zone of inhibition around the disc was recorded and interpreted as susceptible (S), susceptible -dose dependent (S-DD), and resistant (R) as mentioned in Table-1. All disks were obtained from HIMEDIA, India Ltd.

b. Minimum inhibitory concentrations (MIC) method: MIC of amphotericin B, fluconazole and voriconazole against isolated $C$. auris was determined by broth dilution method following the NCCLS approved guideline $\mathrm{M} 27-\mathrm{A} 3$ [18]. All reading was visually taken between 24 and $48 \mathrm{~h}$ of incubation at $35^{\circ} \mathrm{C}$ in aerobic condition and interpreted according to the values mentioned in Table1. Each isolate was tested in duplicate by both disk diffusion and MIC methods.

Table-1: Interpretative breakpoints for $C$. auris by disk diffusion and MICs $(\mu \mathrm{g} / \mathrm{mL})$ methods as per M44-A and M27-A3 CLSI documents

\begin{tabular}{llccc|ccc}
\hline Antifungal agent & Disk content & \multicolumn{3}{c|}{ Zone diameter $(\mathbf{m m})$} & \multicolumn{3}{c}{ MIC $(\boldsymbol{\mu g} / \mathbf{m l})$} \\
\cline { 2 - 7 } & $(\boldsymbol{\mu g})$ & S & S-DD & R & S & S-DD & R \\
\hline AMB & 10 & $\geq 15$ & $10-14$ & $\leq 10$ & $\leq 1.0$ & - & $\geq 2.0$ \\
FLZ & 25 & $\geq 19$ & $15-18$ & $\leq 14$ & $\leq 8.0$ & $16-32$ & $\geq 64$ \\
VOR & 1 & $\geq 17$ & $14-16$ & $\leq 13$ & $\leq 1.0$ & 2.0 & $\geq 4.0$ \\
\hline
\end{tabular}

Note: $A M B=$ amphotericin $B ; F L Z=$ fluconazole; $V O R=$ voriconazole; $S=$ susceptible; $S-D D=$ susceptible dose dependent; $R=$ resistant. 


\section{Results}

Out of 100 Candida sp. tested, 21 isolates were identified as $C$. auris by growth characteristics and carbohydrate assimilation tests. Representative isolates of 21 C. auris, as identified by growth characteristics and carbohydrate assimilation tests were confirmed as $C$. auris by sequencing $(5.8 \mathrm{~S}$ rRNA gene sequences). Sequence analysis of our isolates showed $99 \%-100 \%$ similarity with those of C. auris KP326583, KP131674 and MF167535 5.8S ribosomal RNA gene. Out of 21 isolates, 8 and 13 were isolated from samples of adult and neonate patients respectively (Table-2). Of the 21 C. auris, $14(66.6 \%)$ were isolated from blood samples and the remaining 7 (33.4\%) were from urine samples of adult patients. Except one, all $C$. auris were isolated from blood of neonates admitted in intensive care unit.

The susceptibility pattern of $C$. auris to different antifungal agents by disc diffusion and MIC method is shown in Table-3. Out of 21 C. auris, 14 (66.7\%) were resistant to fluconazole by disk diffusion method. However, out of these 14 resistant isolates 5 were found susceptible dose-dependent (SS-D) by MIC method (Table-3). Most of isolates were sensitive to amphotericin B by both disk diffusion (81.0\%) and MIC (76.2\%) methods. Out of total 21 C. auris tested, $18(85.7 \%)$ was resistant to voriconazole. The detail MIC, $\mathrm{MIC}_{50}$ and $\mathrm{MIC}_{90}$ of all isolates are shown in Table-4.

Table-2: Rate of Isolation of C. auris according to samples and locations $(n=21)$

\begin{tabular}{llc|cc}
\hline \multirow{2}{*}{ Location } & \multicolumn{2}{c|}{ Adult } & \multicolumn{2}{c}{ Neonate } \\
\cline { 2 - 5 } & \multicolumn{4}{c}{ Samples } \\
\cline { 2 - 5 } & Urine & Blood & Urine & Blood \\
\hline ICU & $5(24 \%)$ & $1(5 \%)$ & - & - \\
CCU & $1(5 \%)$ & - & - & - \\
Ward & $1(5 \%)$ & - & - & - \\
NICU & & & & $13(61.9 \%)$ \\
\hline
\end{tabular}

Note: $I C U=$ intensive care unit; $C C U=$ coronary care unit; NICU= neonatal intensive care unit

Table-3: Susceptibility pattern of $C$. auris to amphotericin B, fluconazole and voriconazole by $D D$ and MIC methods

\begin{tabular}{|c|c|c|c|c|c|c|}
\hline \multirow{4}{*}{$\begin{array}{l}\text { Antifungal } \\
\text { agent }\end{array}$} & \multicolumn{6}{|c|}{ Susceptibility by } \\
\hline & \multicolumn{3}{|c|}{ DDM } & \multicolumn{3}{|c|}{ MIC } \\
\hline & $\mathbf{S}$ & S-DD & $\mathbf{R}$ & $\mathbf{S}$ & S-DD & $\mathbf{R}$ \\
\hline & n (\%) & n (\%) & n (\%) & n (\%) & n (\%) & n (\%) \\
\hline AMB & $17(81.0)$ & - & $4(19.0)$ & $16(76.2)$ & - & $5(23.8)$ \\
\hline FLZ & $7(33.3 \%)$ & 0 & $14(66.7)$ & $7(33.3)$ & $5(23.8)$ & $9(42.8)$ \\
\hline VOR & $3(14.3)$ & 0 & $18(85.7)$ & $3(14.3)$ & 0 & 18 (85.7) \\
\hline
\end{tabular}

Note: $A M B=$ amphotericin $B ; F L Z$ = fluconazole; $V O R=$ voriconazole; $S=$ susceptible; $S-D D=$ susceptible dose dependent; $R=$ resistant; $D D M=$ disk diffusion method.

Table-4: MIC of amphotericin B, fluconazole and voriconazole against isolated C. auris $(n=21)$

\begin{tabular}{|c|c|c|c|c|c|c|c|c|c|c|c|c|c|c|}
\hline & \multicolumn{12}{|c|}{ Number of isolate with MIC ( $\mu \mathrm{g} / \mathrm{ml})$} & \multirow[b]{2}{*}{$\mathrm{MIC}_{50}$} & \multirow[b]{2}{*}{$\mathrm{MIC}_{90}$} \\
\hline & .12 & .25 & .5 & 1 & 2 & 4 & 8 & 16 & 32 & 64 & 128 & 256 & & \\
\hline AMB & 0 & 6 & 7 & 3 & 5 & 0 & & & & & & & 0.5 & 2.0 \\
\hline FLZ & 0 & 0 & 0 & 0 & 0 & 0 & 7 & 0 & 5 & 0 & 0 & 9 & 32 & 256 \\
\hline VOR & 1 & 2 & & & & 4 & 10 & 0 & 4 & & & & 8 & 32 \\
\hline
\end{tabular}

Note: $A M B=$ amphotericin $B ; F L Z=$ fluconazole; $V O R=$ voriconazole 


\section{Discussion}

C. auris is an emerging fungus and has become a global nosocomial problem. It causes candidiasis ranging from superficial skin infection to severe invasive bloodstream and multi organs infections. It is variably resistant to multiple antifungal drugs commonly used to treat Candida infections. C. auris was first isolated from the ear canal of a 70year-old Japanese woman in Japan in 2009 [4]. In 2011, the first three cases of disease-causing $C$. auris were reported from South Korea [19]. The first report of a $C$. auris outbreak in Europe was in 2016 [20]. Up till 2019, C. auris in clinical samples has been documented in more than 30 countries of the world [21].

This is the first study of $C$. auris in Bangladesh. The study has revealed the presence of $C$. auris infection in the hospitalized patients of Bangladesh. About $62 \%$ and $29 \%$ of $C$. auris were found in pediatric and ICU adult patients respectively and most frequently it is isolated from blood (67\%). A recent study in USA documented 77 clinical cases of $C$. auris from seven states of which 45 were bloodstream isolates and the remaining were from urine $(n=11)$, respiratory tract $(n=8)$, bile fluid (4), wound (4), CVC tip (2), bone, ear and jejunal biopsy specimens [14].

Antifungal susceptibility of Candida species varies from place to place and species to species. Susceptibility of $C$. auris to fluconazole, voriconazole and amphotericin B found in the present study was similar to the findings of other studies reported from different countries of the world $[11,12,21]$.

In the present study, 5 (23\%) C. auris found resistant by disk diffusion method to fluconazole were actually dose dependent susceptible by MIC method. These $C$. auris isolates exhibited slightly hazy zone of growth within the zone of inhibition in disk diffusion method and were recorded as 'resistant' by disk diffusion test. Therefore, any strain showing hazy zone of growth within the zone of inhibition should be confirmed by MIC method as higher dose of fluconazole could be used to treat infection by such S-DD strains of $C$. auris. It is important because fluconazole is a cheaper drug compared to other more expensive and toxic antifungal agents.
In our study, almost all (20/21) C. auris were isolated from patients admitted either in adult or neonatal intensive care units of the hospital. The finding of the study emphasizes the need for quick detection of this organism in clinical samples to prevent its spread in the hospitals. So, special attentions are needed to quickly detect $C$. auris and its antifungal susceptibility for appropriate treatment and for the prevention of its nosocomial transmission. Present method of identifying $C$. auris by biochemical and sugar assimilation tests is time consuming and is often fraught with difficulties. Rapid technique is needed for quick diagnosis of $C$. auris infection to initiate timely and appropriate treatment. Further study is warranted to determine its true magnitude in the hospitals of Bangladesh. Also, measures should be taken to create awareness among the microbiologists and clinicians regarding the importance of $C$. auris infection in severely ill patients requiring long hospital stay or admission in intensive care units.

\section{References}

1. Douglas L. Candida biofilms and their role in infection. Trends in Microbiol.2003; 11(1): 30-36.

2. Richter SS, Galask RP, Messer SA, Hollis RJ, Diekema DJ and Pfaller MA. Antifungal susceptibilities of Candida species causing vulvo-vaginitis and epidemiology of recurrent cases. J Clin Microbiol. 2005; 43(5): 2155-2162.

3. Deorukhkar S. Saini S. Non albicans Candida species: its isolation pattern, species distribution, virulence factors and antifungal susceptibility profile. Int J Med Sci Public Health. 2013; 2(3): 533-538.

4. Satoh K, Makimura K, Hasumi Y, Nishiyama Y, Uchida K, Yamaguchi H. Candida auris sp. Nov., a novel ascomycetous yeast isolated from the external ear canal of an inpatient in a Japanese hospital. Microbiol Immunol. 2009; 53: 41-44.

5. Jeffery-Smith A, Taori SK, Schelenz S, Jeffery K, Johnson EM, Borman A, Candida auris Incident Management Team, Rohini Manuel, Colin S. Brown. Candida auris: a review of the literature. Clin Microbiol Rev. 2018; 31(1): e00029-17. 
6. Osei Sekyere J. Candida auris: A systematic review and meta-analysis of current updates on an emerging multidrug-resistant pathogen. Microbiologyopen. 2018; 7(4): e00578.

7. Calvo B, Melo AS, Perozo-Mena A, Hernandez $M$, Francisco EC, Hagen $F$, et al. First report of Candida auris in America: clinical and microbiological aspects of 18 episodes of candidemia. J Infect. 2016; 73(4): 369-74.

8. Rozwadowski F, McAteer J, Chow NA, Skrobarcek K, Forsberg K, Barrett PM, et al. Prevalence and risk factors for Candida auris colonization among patients in a long term acute care hospital - New Jersey, 2017. Open Forum Infect Dis. 2018; 5(Suppl 1): S14.

9. Chowdhary A, Sharma C, Duggal S, Agarwal K, Prakash A, Singh PK, et al. New clonal strain of Candida auris, Delhi, India. Emerg Infect Dis. 2013; 19(10): 1670-3.

10. Ben-Ami R, Berman J, Novikov A, Bash E, Shachor-Meyouhas Y, Zakin S, et al. Multidrugresistant Candida haemulonii and $C$. auris, Tel Aviv, Israel. Emerg Infect Dis. 2017; 23(1): 10.

11. Chowdhary et al. Multidrug-resistant endemic clonal strain of Candida auris in India. Eur J Clin Microbiol Infect Dis. 2014; 33: 919-26.

12. Lockhart SR, Etienne KA, Vallabhaneni S, Farooqi J, Chowdhary A, Govender NP, Colombo AL, Calvo B, Cuomo CA. Simultaneous emergence of multidrug-resistant Candida auris on 3 continents confirmed by whole-genome sequencing and epidemiological analyses. Clin Infect Dis. 2017; 64 (2): 134-140.

13. Chowdhary A, Prakash A, Sharma C, Kordalewska M, Kumar A, Sarma S, et al. A multicentre study of antifungal susceptibility patterns among 350 Candida auris isolates (2009-17) in India: role of the ERG11 and FKS1 genes in azole and echinocandin resistance. $J$ Antimicrob Chemother. 2018; 73: 891-899.
14. Borman AM, Szekely A, Johnson EM. Comparative pathogenicity of United Kingdom isolates of the emerging pathogen Candida auris and other key pathogenic Candida species. mSphere 2016; 1(4):e00189-16.

15. Adams ED, Cooper BH. Evaluation of a modified Wickerham medium for identifying medically important yeasts. Am J Med Technol. 1974; 40(9): 377-388.

16. Fujita SI, Senda Y, Nakaguchi S, Hashimoto T. Multiplex PCR using internal transcribed spacer 1 and 2 regions for rapid detection and identification of yeast strains. J Clin Microbiol. 2001; 39 (10): 3617-3622.

17. Methods for antifungal disk diffusion susceptibility testing of yeasts; Approved Guideline M44-A. Vol: 29, Number 17. 2nd ed. Wayne, PA: National Committee for Clinical Laboratory Standards; 2004.

18. Reference method for broth dilution antifungal susceptibility testing of yeasts M27-A3. Vol: 28, Number. 14. 3rd ed. Wayne, PA: Clinical and Laboratory Standards Institute; 2008.

19. Lee WG, Shin JH, Uh Y, Kang MG, Kim SH, Park $\mathrm{KH}$, et al. First three reported cases of nosocomial fungemia caused by Candida auris. J Clin Microbiol. 2011; 49 (9): 3139-3142.

20. Schelenz S, Hagen F, Rhodes JL, Abdolrasouli A, Chowdhary A, Hall A, et al. First hospital outbreak of the globally emerging Candida auris in a European hospital. Antimicrob Resist Infect Control. 2016; 5: 35.

21. Tracking Candida auris, July 12, 2019. Center for Disease Control and Prevention, Atalanta, GA, USA. www.cdc.gov/fungal/candida-auris/ tracking-c-auris.html 